Akil J. Harfash* and Huda A. Challoob

\title{
Nonhomogeneous porosity and thermal diffusivity effects on stability and instability of double-diffusive convection in a porous medium layer: Brinkman Model
}

https://doi.org/10.1515/nleng-2018-2001

Received February 4, 2017; revised June 24, 2017; accepted December 5, 2017.

\begin{abstract}
A model for double-diffusive convection in anisotropic and inhomogeneous porous media has been analysed. In particular, the effect of variable permeability and thermal diffusivity has been studied using the Brinkman model. Moreover, we analyse the effect of slip boundary conditions on the stability of the model. Due to numerous applications in micro-electro-mechanicalsystems (MEMS) and other microfluidic devices, such a study is essential to have. Both linear instability analysis and nonlinear stability analysis are employed. We accurately analyse when stability and instability will commence and determine the critical Rayleigh number as a function of the slip coefficient.
\end{abstract}

Keywords: Double-diffusive; variable permeability; variable thermal diffusivity; slip boundary conditions; Brinkman model

\section{Introduction}

Double diffusive convection in porous media has been a focus for researchers, since it has applications in a number of areas, including geophysics, the enhanced recovery of petroleum reservoirs, the underground diffusion of chemical wastes, seabed hydrodynamics, and crystal growth, for more details see [1], [2] and the references therein.

The critical Rayleigh number of the natural convection in anisotropic porous layers was analyzed by [3]

\footnotetext{
*Corresponding Author: Akil J. Harfash, Department of Mathematics, College of Sciences, University of Basrah, Basrah, Iraq, E-mail: akilharfash@gmail.com

Huda A. Challoob, Department of Mathematics, College of Sciences, University of Basrah, Basrah, Iraq
}

and [4] where there was anisotropy in permeability and anisotropy both in permeability and thermal diffusivity, respectively. [5] proposed a general method of treating this kind of problem, and they reported results of numerical calculation of natural convection heat transfer near the critical Rayleigh number, using the Galerkin method. They also compared the calculated Nusselt number with the experimental values. All of these studies cited above assumed Darcy's law. One of the recent and important study is introduced by [6]. In this study, the critical Rayleigh number at the onset of natural convection was studied by linear stability analysis for high porosity anisotropic horizontal porous layers of uniformly arraying vertical thin circular wires stretched across a hot and a cold surface. Navier-Stokes equations including flow resistance by the wires were solved since Darcy's law cannot be applied due to high porosity.

Capone et al. [7] analyzed the onset of convection in a horizontal fluid-saturated porous layer uniformly heated from below in which the permeability and thermal diffusivity vary linearly or exponentially in the vertical direction. They studied this problem with constant gravity. However, Harfash [8] studied this problem with variable gravity with respect to the vertical direction and tested the validity of both the linear instability and global nonlinear energy stability thresholds for this problem using a three-dimensional simulation. Moreover, a model for double-diffusive convection in anisotropic and inhomogeneous porous media using Darcy's law has been analysed in [9]. The effects of variable permeability, thermal diffusivity and variable gravity with respect to the vertical direction, have been studied in [9].

There is increasing interest in micro-electromechanical-systems (MEMS), and flow in microfluidic channels due to their applications in the electronics and related industries. In particular, at nanoscales there is increasing evidence that boundary conditions of slip type are needed rather than those of no-slip, cf. Cercignani [10], Duan [11], Duan \& Muzychka [12], Lauga et 
al. [13], Morini et al. [14], Priezjev [15], Zhang et al. [16], Zhang et al. [17]. An especially important application of microscale flow involving slip boundary conditions is to flow in porous metallic foams. Lefebvre et al. [18] give many industrial examples of this and provide a thorough review of the state of the art. The object of this paper is to investigate a double diffusive convection problem in an inhomogeneous Brinkman porous media. In particular, the effect of variable permeability, thermal diffusivity and slip boundary conditions, has been studied.

Microchannels heat sinks with pin fins or ribs are considered as porous media. If the distance between the solid materials in micro dimensions, the slip is obtained in the boundaries (Knudsen number is appareled) for the flow of gases in the pores. The Knudsen number $(\mathrm{Kn})$ is a dimensionless number defined as the ratio of the molecular mean free path length to a representative physical length scale. This length scale could be, for example, the radius of a body in a fluid. Knudsen number is expressed by the following equation.

$$
K n=\lambda / D_{h},
$$

where $K n$ Knudsen number, $\lambda$ mean free path $(m), D_{h}$ hydraulic diameter $(m)$.

The mean free path of a particle, such as a molecule, is the average distance the particle travels between collisions with other particles. The mean free path is a function of temperature and pressure and is defined as follows

$$
\lambda=\frac{K_{b} T}{\sqrt{2} \pi P d^{2}},
$$

where $K_{b}, T, P$, and $d$ are the Boltzmann constant $1.3806503 E-23\left(\mathrm{~m}^{2} \mathrm{kgs}^{-2} \mathrm{~K}^{-1}\right)$, temperature $(K)$, pressure $\left(\mathrm{N} / \mathrm{m}^{2}\right)$, and molecular diameter $(\mathrm{m})$.

As Knudsen number increases, rarefaction effects become more important and continuum approach breaks down. However, it is convenient to differentiate the flow regimes in function of $K n$, and the following classification:

- $\quad$ For $K n<10^{-3}$, the flow is a continuum flow and it is accurately modeled by the compressible NavierStokes equations with classical no-slip boundary conditions.

- For $10^{-3}<K n<10^{-1}$, the flow is a slip flow and the Navier-Stokes equations remain applicable, provided a velocity slip and a temperature jump are taken into account at the walls. These new boundary conditions point out that rarefaction effects become sensitive at the wall first.

- For $10^{-1}<K n<10$, the flow is a transition flow and the continuum approach of the Navier-Stokes equa- tions is no longer valid. However, the intermolecular collisions are not yet negligible.

- For $K n>10$, the flow is a free molecular flow and the occurrence of intermolecular collisions is negligible compared with the one of collisions between the gas molecules and the walls.

In the gas reservoir (petroleum reservoirs), there are a difference between the pressures (temperature) of reservoir layers which makes the diffusion is transfer from high to low through the pores, where the most of pores are in the micro dimension or less which produces a slip in the boundaries.

The really interesting situation from both a geophysical and a mathematical viewpoint arises when the layer is simultaneously heated from below and salted from below. In this situation heating expands the fluid at the bottom of the layer and this in turn wants to rise thereby encouraging motion due to thermal convection. On the other hand, the heavier salt at the lower part of the layer has exactly the opposite effect and this acts to prevent motion through convective overturning. Thus, these two physical effects are competing against each other. Due to this competition, it means that the linear theory of instability does not always capture the physics of instability completely and (sub-critical) instabilities may arise before the linear threshold is reached. Due to the possibility of sub-critical instabilities occurring, it is very important to test the accuracy of the linear instability threshold in capturing the onset of the instability of the double diffusive problem when the layer is heated and salted from below. Recent contributions on the study of convective instabilities in fluid and porous media include [19-28]. In the last few years and via a new methodology which is called the auxiliary system method, Rionero proved the coincidence between linear and nonlinear stability thresholds for the convection problem in many real situations (for more details, see [2934]).

The plan of the paper is as follows. In the next section, we present the governing equations of motion and derive the associated perturbation equations. Then, we perform linear instability analysis (Section 3) and global nonlinear stability analysis (Section 4) and derive numerically the instability/stability thresholds. Since the stability analyses involve eigenvalue problems with non-constant coefficients these problems must be solved numerically and a suitable numerical method is described in section 5 . In Section 6, the numerical results for the linear theory and a direct comparison with those of the global nonlinear theories are presented. 


\section{Mathematical formulation and governing equations}

Let us consider a layer of a porous medium bounded by two horizontal planes and saturated by a binary fluid mixture. Let $d>0, \Omega=R^{2} \times(0, d)$ and $O x y z$ be a cartesian frame of reference with unit vectors $i, j, k$, respectively. We assume that the Oberbeck-Boussinesq approximation be valid and that the flow in the porous medium is governed by Darcy's law with the variable permeability $\varphi(z)=$ $k_{0} \hat{f}(z) \geq 0, \hat{f}(z) \in C^{0}[0, d] \cup C^{1}(0, d)$ and the thermal diffusivity $K(z)=K_{0} \hat{g}(z) \geq 0, \hat{g}(z) \in C^{0}[0, d] \cup C^{2}(0, d)$, $\mathbf{k}=(0,0,1)$, the basic equations are:

$$
\begin{gathered}
\tilde{\vartheta} v_{i, t}=-\frac{\mu}{\varphi(z)} v_{i}-P_{, i}-k_{i} \mathrm{~g} \rho(T, C)+\tilde{\chi} \Delta v_{i}, \\
v_{i, i}=0, \\
T_{, t}+v_{i} T_{, i}=\left[K(z) T_{, j}\right]_{, j}, \\
\tilde{\varepsilon} C_{, t}+v_{i} C_{, i}=\kappa_{C} \Delta C,
\end{gathered}
$$

where (2.2) is the incompressibility condition and (2.3) and (2.4) are the equations of energy and solute balance, respectively. The derivation of equations (2.1) - (2.4) may be found in $[1,2]$.

We have denoted $\mathbf{v}, P, T, C, \tilde{\varepsilon}, \mathrm{g}, \tilde{\chi}, \tilde{\vartheta}$ and $\kappa_{c}$ to be the velocity, pressure, temperature, concentration of salt, porosity, gravitational acceleration, the Brinkman coefficient (or effective viscosity), inertia coefficient and salt diffusivity, respectively. The density $\rho$ is of the form

$$
\rho(T, C)=\rho_{0}\left(1-\alpha_{t}\left(T-T_{0}\right)+\alpha_{c}\left(C-C_{0}\right)\right)
$$

where $\rho_{0}, T_{0}$ and $C_{0}$ are a reference density, temperature and concentration, respectively, and $\alpha_{t}$ and $\alpha_{c}$ are the coefficients for thermal and solute expansion, respectively. Throughout, we use standard indicial notation and the Einstein summation convention so that e.g. $v_{i, t}=\partial v_{i} / \partial t$, and $P,{ }_{i}=\partial P / \partial x_{i}$, and $\Delta$ is the Laplacian.

Alongside equations (2.1)-(2.4) we must prescribe boundary conditions on the fluid at $\partial \Omega$. The classical approach is to state that fluid molecules adjacent to a solid surface are at rest, with respect to that surface. This is called the no-slip boundary condition, and despite its historical prevalence in the fluid mechanics literature, it is an assumption that cannot be derived from first principles, $[13,35]$.

Boundary slip (that is, motion of a fluid with respect to a solid surface) in gases was predicted by Maxwell [36], and experiments have shown that gas flow in geometries with dimensions of the order of the mean free path of the gas can show significant slip at a boundary, see [37]. However, the measurement of boundary slip of Newtonian liquids has been the subject of much more research, see [3840]. In particular, there is growing evidence that fluid velocity at the boundary is dependent upon the shear strain, see for example Craig et al. [38, 39] and Zhu \& Granick [40].

Navier [41] proposed a linear boundary condition relating $\mathbf{v}$ to the shear rate, which has become standard in the study of boundary slip problems. Letting the surface $\partial \Omega$ have unit normal $n(x)$ directed out of the fluid, and $\hat{t}(x)$ be either of the vectors tangent to $\partial \Omega$ at $x \in \partial \Omega$, this boundary condition can be expressed as

$$
\begin{gathered}
\left.v_{i} n_{i}\right|_{\partial \Omega}=V_{i} n_{i},\left.\right|_{\partial \Omega} \\
\left.v_{i} \hat{t}_{i}\right|_{\partial \Omega}=\left(V_{i}-\tilde{\lambda} \epsilon_{i j} n_{j}\right) \hat{t}_{i},\left.\right|_{\partial \Omega}
\end{gathered}
$$

where $\epsilon=\epsilon(\mathbf{v})$ is the shear strain tensor, and $V_{i}=V_{i}(\partial \Omega)$ is the $i$ th component of the local surface velocity. The model is essentially to set the component of $\mathbf{v}$ normal to $\partial \Omega$ to be zero, thus imposing a condition of zero flux across the surface, while setting the two tangential components of $\mathbf{v}$ proportional to the corresponding components of shear stress. We denote the constant of proportionality $\tilde{\lambda} \geq 0$, which has the dimension of length, and it can be seen that $\tilde{\lambda}=0$ in (2.5) and (2.6) recovers the no-slip boundary condition. Clearly, if we wish to perform any numerical work on the system (1.1)-(1.4) we must have a value for $\tilde{\lambda}$ at hand.

We now apply the boundary conditions (2.5)-(2.6) to the Boussinesq model. Since the fluid is confined to $\Omega$, from (2.5) we impose,

$$
v_{3}=0, \quad \text { at } \quad z=0, d,
$$

and we note that since there is no variation of $v_{3}$ in the upper surface $\partial \Omega_{L}$ and the lower surface $\partial \Omega_{U}$, we must have

$$
v_{3, x}=v_{3, y}=0, \quad \text { at } \quad z=0, d .
$$

Let $\lambda_{L}$ be the slip length associated with the fluid-solid interface at $\partial \Omega_{L}$, and define $\lambda_{U}$ similarly. Then, from (2.6) we have

$v_{1}-\lambda_{L} v_{1, z}=0, \quad v_{2}-\lambda_{L} v_{2, z}=0, \quad$ at $\quad z=0$,

$v_{1}+\lambda_{U} v_{1, z}=0, \quad v_{2}+\lambda_{U} v_{2, z}=0, \quad$ at $\quad z=d$

We note that these boundary conditions allow the zero solution $\mathbf{v}=0$, which represents a fluid at rest. 
Applications of the above model can appear in the following fields:

- Agricultural applications: e.g. fermentation process in food industries, freeze drying of food products, grain storage, soil heating to increase the growing season.

- Environmental applications: e.g. ground water pollution, ground water systems, storage of radioactive waste, water movement in geothermal reservoirs.

- Industrial applications: e.g. artificial freezing of ground as a structural support and as a water barrier for construction and mining purposes, crude oil production and recovery systems, porous radiant burners (PRBs), post accident heat removal (PAHR), solidification of castings, study of heat transfer phenomenon of buried electrical cables and transformer cables, fluidized bed combustion.

- Thermal conversion and storage systems: e.g. catalytic reactors, geothermal systems, packed beds, fluidized bed, heat pipes, sensible, latent and thermochemical energy storage systems.

The temperature and concentration boundary conditions for the problem are $T=T_{U}$ and $C=C_{U}$ at $z=d$ and $T=T_{L}$ and $C=C_{L}$ at $z=0$, where $T_{L}>T_{U}$ and $C_{L}>C_{U}$, so that the system is being heated and salted from below. Under these conditions, the conduction solution in whose stability we are interested is

$\bar{v}_{i} \equiv 0$,

$$
\bar{C}=C_{L^{-}}-\frac{\left(C_{L}-C_{U}\right)}{d} z,
$$

$\bar{T}=\frac{\left(T_{U}-T_{L}\right)}{\int_{0}^{d} \frac{d \mathfrak{F}}{\hat{g}(\mathfrak{F})}} \int_{0}^{z} \frac{d \mathfrak{F}}{\hat{g}(\mathfrak{F})}+T_{L}, \quad \frac{d \bar{P}}{d z}=-\rho \mathrm{g} \alpha_{c} \bar{C}-\rho \mathrm{g} \alpha \bar{T}$,

To investigate the stability of these solutions, we introduce perturbations $\left(u_{i}, p, \theta, \phi\right)$ by

$$
v_{i}=u_{i}+\bar{v}_{i}, \quad P=p+\bar{P}, \quad T=\theta+\bar{T}, \quad C=\phi+\bar{C} .
$$

The perturbation equations are nondimensionalized according to the scales (stars denote dimensionless quantities)

$$
\begin{gathered}
p=\mathcal{P} p^{\star}, \quad \mathcal{P}=\frac{\mu K_{0}}{k_{0}}, \quad \theta=\theta^{\star} \sqrt{\frac{d \beta \mu K_{0}}{\mathrm{~g} \rho_{0} \alpha_{t} k_{0} \bar{g}}}, \quad x_{i}=d x_{i}^{\star}, \\
\phi=\phi^{\star} \sqrt{\frac{\mu K_{0}\left(C_{L}-C_{U}\right)}{\mathrm{g} \rho_{0} \alpha_{c} k_{0} d}}, u_{i}=\frac{K_{0}}{d} u_{i}^{\star}, t=\frac{d^{2}}{K_{0}} t^{\star}, L e=\frac{K_{0}}{\kappa_{c}}, \\
R_{t}^{2}=\frac{\mathrm{g} \rho_{0} \alpha_{t} k_{0} d^{3} \beta}{\mu K_{0} \bar{g}}, \quad \beta=\frac{T_{L}-T_{U}}{d}, \quad f\left(z^{\star}\right)=\hat{f}\left(d z^{\star}\right),
\end{gathered}
$$

$g\left(z^{\star}\right)=\hat{g}\left(d z^{\star}\right), \quad R_{c}^{2}=\frac{\mathrm{g} \rho_{0} \alpha_{c} k_{0} d\left(C_{L}-C_{U}\right)}{\mu K_{0}}, \bar{g}=\int_{0}^{d} \frac{d \mathfrak{F}}{g(\mathfrak{F})}$,

where $R_{t}^{2}$ and $R_{c}^{2}$ are the thermal and solute Rayleigh numbers, respectively. The dimensionless perturbation equations are (after omitting all stars)

$$
\begin{gathered}
\vartheta u_{i, t}=-\frac{1}{f(z)} u_{i}-p_{, i}+R_{t} \theta k_{i}-R_{c} \phi k_{i}+\chi \Delta u_{i}, \\
u_{i, i}=0, \\
\theta, t+u_{i} \theta_{, i}=R_{t} \frac{1}{g(z)} w+g(z) \Delta \theta+g^{\prime}(z) \theta_{, 3}, \\
\tilde{\varepsilon} \phi, t+u_{i} \phi_{, i}=R_{c} w+\frac{1}{L e} \Delta \phi .
\end{gathered}
$$

where $\vartheta$ and $\chi$ are non-dimensional equivalents of $\tilde{\vartheta}$ and $\tilde{\chi}$, respectively. In these equations, $R_{t}$ is the Rayleigh number, $w=u_{3}, f(z), g(z)$ are non-dimensionalized form of variable permeability and the thermal diffusivity functions, respectively. These equations hold on $\{z \in(0,1)\} \times\{(x, y) \in$ $\left.\mathbb{R}^{2}\right\}$. In (2.11), for variable permeability and thermal diffusivity, we will address as exponential porous media such that $f(z)=e^{A z}$, and $g(z)=e^{B z}$. Since the fluid is incompressible we have that $w_{, z}=-\left(u_{, x}+v, y\right)$. Therefore $w_{, z z}=-(u, x z+v, y z)$, and from the boundary conditions on $u$ and $v$ we see that $w$ satisfies

$$
w=0, \quad w_{, z}=N_{L} w_{, z z} \quad \text { at } \quad z=0,
$$

$$
w=0, \quad w_{, z}=-N_{U} w_{, z z} \quad \text { at } \quad z=1,
$$

while, $\theta$ and $\phi$ vanish at $z=0$ and $z=1$. In (2.12) and (2.13), $N_{U}$ and $N_{L}$ are dimensionless parameters of $\lambda_{U}$ and $\lambda_{L}$, respectively. Also, $u_{i}, p, \theta$ and $\phi$ are assumed periodic in the $x$ and $y$ directions.

\section{Linear instability}

In order to study linear instability, we discard the nonlinear terms in (2.11). A time dependence such as $u_{i}=$ $e^{\sigma t} u_{i}(\mathbf{x}), \pi=e^{\sigma t} \pi(\mathbf{x}), \theta=e^{\sigma t} \theta(\mathbf{x}), \phi=e^{\sigma t} \phi(\mathbf{x})$ is now assumed and then, after removing, the pressure perturbation the linearized instability equations that arise from (2.11) are found to be

$$
\begin{gathered}
\sigma \vartheta \Delta w=-\frac{1}{f(z)} \Delta w+\frac{f^{\prime}(z)}{f^{2}(z)} w_{, z}+R_{t} \Delta^{\star} \theta-R_{c} \Delta^{\star} \phi+\chi \Delta^{2} w \\
\sigma \theta=R_{t} \frac{1}{g(z)} w+g(z) \Delta \theta+g^{\prime}(z) \theta_{, 3}
\end{gathered}
$$




$$
\tilde{\varepsilon} \sigma \phi=L e^{-1} \Delta \phi+R_{c} w .
$$

where $\Delta^{\star}$ is the horizontal Laplacian $\Delta^{\star}=\partial^{2} / \partial x^{2}+$ $\partial^{2} / \partial y^{2}, D=d / d z$, and (3.1) hold on $\mathbb{R}^{2} \times(0,1)$. To proceed from equations (3.1), a plane tiling form $h(x, y)$ is introduced, see e.g. [42, 43]), and then we put $w=W(z) h(x, y)$, $\theta=\Theta(z) h(x, y)$ and $\phi=\Phi(z) h(x, y)$ and introduce the wavenumber $a$ by $\Delta^{\star} h=-a^{2} h$. Equations (3.1) then yield the eigenvalue problem

$$
\begin{gathered}
\chi f^{2}(z)\left(D^{2}-a^{2}\right)^{2} W-f(z)\left(D^{2}-a^{2}\right) W+f^{\prime}(z) D W \\
-a^{2} R_{t} f^{2}(z) \Theta+a^{2} R_{c} f^{2}(z) \Phi=9 \sigma\left(D^{2}-a^{2}\right) W, \\
g(z)\left(D^{2}-a^{2}\right) \Theta+g^{\prime}(z) D \Theta+R \frac{1}{g(z)} W=\sigma \Theta, \\
L e^{-1}\left(D^{2}-a^{2}\right) \Phi+R_{c} W=\tilde{\varepsilon} \sigma \Phi,
\end{gathered}
$$

In (3.4), $z \in(0,1)$, and the boundary conditions are

$$
\begin{aligned}
& \Theta=\Phi=W=D W-N_{L} D^{2} W=0, \quad \text { at } \quad z=0, \\
& \Theta=\Phi=W=D W+N_{U} D^{2} W=0, \quad \text { at } \quad z=1 .
\end{aligned}
$$

Detailed numerical results are presented in Section 7.

\section{Nonlinear energy stability theory}

When adopting a linear analysis approach, the perturbation to the steady state is assumed to be small, and so nonlinear terms in the governing set of partial differential equations are discarded. It has been proved that linear analysis often provides little information on the behavior of the nonlinear system [42], so in such cases only instability can be deduced from the linear thresholds, as any potential growth in the nonlinear terms is not considered.

Let $V$ be a period cell for a disturbance to (2.11), and let $\|\cdot\|$ and $\langle\cdot, \cdot\rangle$ be the norm and inner product on $L^{2}(V)$. Next, multiply $(2.11)_{1},(2.11)_{3}$ and (2.11) $)_{4}$ by $u_{i}, \theta$ and $\phi$ and integrating over $V$. By introducing a coupling parameters $\lambda_{1}, \lambda_{2}>0$ we may then derive

$$
\frac{d E}{d t}=\mathcal{J}-\mathcal{D},
$$

where the functions $E$ and $I$ are given by

$$
E(t)=\frac{\lambda_{1}}{2}\|\theta\|^{2}+\tilde{\varepsilon} \frac{\lambda_{2}}{2}\|\phi\|^{2}+\frac{\vartheta}{2}\|\mathbf{u}\|^{2},
$$

and

$$
\mathcal{J}=R_{t}\left\langle\Psi_{1}(z) \theta, w\right\rangle-R_{c}\left\langle\Psi_{2}(z) \theta, w\right\rangle,
$$

with the dissipation $D$ being defined by

$$
\mathcal{D}=\lambda_{1}\left\langle g(z)|\nabla \theta|^{2}\right\rangle+\left\langle\frac{1}{f(z)}|\mathbf{u}|^{2}\right\rangle+\lambda_{2} L e^{-1}\|\nabla \phi\|^{2}
$$

$$
+\chi\|\nabla \mathbf{u}\|^{2}+\frac{1}{N_{L}} \int_{\partial \Omega_{L}}|\mathbf{u}|^{2} d S+\frac{1}{N_{U}} \int_{\partial \Omega_{U}}|\mathbf{u}|^{2} d S,
$$

where $d S$ is a surface element, $\Psi_{1}(z)=1+\frac{\lambda_{1}}{g(z)}, \Psi_{2}=1-\lambda_{2}$ and $\mathbf{u}$ is explicitly written as $\mathbf{u}=(u, v, w)$. Define $R_{E}$ by

$$
\frac{1}{R_{E}}=\max _{\mathcal{H}} \frac{\mathcal{J}}{\mathcal{D}}
$$

where $H$ is the space of admissible functions, i.e. $u_{i}, \theta, \phi \in$ $H^{1}(V)$ with $u_{i}$ solenoidal and $u_{i}, \theta, \phi$ satisfying the boundary conditions. Then from (4.1) we derive

$$
\frac{d E}{d t} \leq-\mathcal{D}\left(1-\frac{1}{R_{E}}\right) .
$$

Poincare's inequality ensures that there is a constant $c>$ 0 such that $\mathcal{D}>c E$ and then if $R_{E}>1$, one may show from inequality (4.6) that $E$ decays exponentially and we have global nonlinear stability, i.e. for all initial data. The nonlinear stability threshold then requires the solution of (4.5). The Euler-Lagrange equations which arise from (4.5) are

$$
\begin{gathered}
2 \chi \Delta u_{i}-\frac{2}{f} u_{i}+R_{t} k_{i} \Psi_{1} \theta-R_{c} k_{i} \Psi_{2} \phi=\zeta_{, i}, \\
R_{t} \frac{\Psi_{1}}{g} w+2 \lambda_{1} \Delta \theta+2 \lambda_{1} \frac{g^{\prime}}{g} \theta_{, 3}=0, \\
2 \lambda_{2} L e^{-1} \Delta \phi-R_{c} \Psi_{2} w=0,
\end{gathered}
$$

where $\zeta$ is a Lagrange multiplier. To remove the Lagrange multiplier we take the third component of the double curl of $(4.5)_{1}$, and introducing the normal mode representation and notation as presented in section 3 , thus (4.5) then becomes

$$
\begin{aligned}
& 2 \chi f^{2}(z)\left(D^{2}-a^{2}\right)^{2} W-2 f\left(D^{2}-a^{2}\right) W-2 D f D W \\
& +a^{2} R_{c} f^{2} \Psi_{2} \Phi=a^{2} R_{t} f^{2} \Psi_{1} \Theta, \\
& 2 g \lambda_{1}\left(D^{2}-a^{2}\right) \Theta+2 \lambda_{1} g^{\prime} D \Theta+R_{t} \Psi_{1} W=0, \\
& 2 \lambda_{2} L e^{-1}\left(D^{2}-a^{2}\right) \Phi-R_{c} \Psi_{2} W=0,
\end{aligned}
$$

together with boundary conditions (3.5). Numerical results are presented in Section 7 .

\section{Numerical technique}

In this section, we use the Chebyshev collocation method to solve the eigenvalue system (3.4) and (4.8). In the Chebyshev collocation method, system (3.4) is rewritten in terms of second and third order derivatives only. Letting $\Pi=$ $D W$, (3.4) can be expressed as the four 2 nd order equations. The system is then transformed onto the Chebyshev 
domain $(-1,1)$ and the solutions $W$ and $\theta$ treated as independent variables and expanded in a series of Chebyshev polynomials

$$
\begin{aligned}
W & =\sum_{n=0}^{N} w_{n} T_{n}(z), \\
\Pi & =\sum_{n=0}^{N} \Pi_{n} T_{n}(z), \\
\Theta & =\sum_{n=0}^{N} \Theta_{n} T_{n}(z), \Phi=\sum_{n=0}^{N} \Phi_{n} T_{n}(z),
\end{aligned}
$$

then, we insert (5.1) into the equations (3.4), and then substitute the Gauss-Labatto points which are defined by

$$
y_{i}=\cos \left(\frac{\pi i}{N-3}\right), \quad i=0, \ldots, N-2 .
$$

Thus, we obtain $4 N-4$ algebraic equations for $4 N+4$ unknowns $W_{0}, \ldots, W_{N}, \Pi_{0}, \ldots, \Pi_{N}, \Theta_{0}, \ldots, \Theta_{N}, \Phi_{0}, \ldots, \Phi_{N}$. Now, we can add six rows using the boundary conditions (3.5) as follows

$$
\begin{gathered}
B C_{1}: \sum_{n=0}^{N} W_{n}=0, \\
B C_{2}: \sum_{n=0}^{N}(-1)^{n} W_{n}=0, \\
B C_{3}: \sum_{n=0}^{N} n^{2} N_{U} \Pi_{n}+\Pi_{n}=0, \\
B C_{4}: \sum_{n=0}^{N}(-1)^{n+1} n^{2} N_{U} \Pi_{n}-(-1)^{n} \Pi_{n}=0, \\
B C_{5}: \sum_{n=0}^{N} \Theta_{n}=0, \\
B C_{6}: \sum_{n=0}^{N}(-1)^{n} \Theta_{n}=0, \quad B C_{7}: \sum_{n=0}^{N} \Phi_{n}=0, \\
B C_{8}: \sum_{n=0}^{N}(-1)^{n} \Phi_{n}=0 .
\end{gathered}
$$

The inner product of each equation is taken with some $T_{k}$ and the orthogonality of the Chebyshev polynomials exploited to obtain the following generalised eigenvalue problem:

$$
\begin{aligned}
& \left(\begin{array}{cccc}
2 D & -I & O & O \\
B C_{1} & 0 \ldots 0 & 0 \ldots 0 & 0 \ldots 0 \\
B C_{2} & 0 \ldots 0 & 0 \ldots 0 & 0 \ldots 0 \\
\Omega_{0} & \Omega_{1} & -a^{2} R_{t} \Sigma & a^{2} R_{c} \Sigma \\
0 \ldots 0 & B C_{3} & 0 \ldots 0 & 0 \ldots 0 \\
0 \ldots 0 & B C_{4} & 0 \ldots 0 & 0 \ldots 0 \\
R_{t} Y & O & \Omega_{2} & O \\
0 \ldots 0 & 0 \ldots 0 & B C_{5} & 0 \ldots 0 \\
0 \ldots 0 & 0 \ldots 0 & B C_{6} & 0 \ldots 0 \\
R_{c} I & O & O & L e^{-1} \Omega_{3} \\
0 \ldots 0 & 0 \ldots 0 & 0 \ldots 0 & B C_{7} \\
0 \ldots 0 & 0 \ldots 0 & 0 \ldots 0 & B C_{8}
\end{array}\right) X \\
& =\sigma\left(\begin{array}{cccc}
O & O & O & 0 \\
0 \ldots 0 & 0 \ldots 0 & 0 \ldots 0 & 0 \ldots 0 \\
0 \ldots 0 & 0 \ldots 0 & 0 \ldots 0 & 0 \ldots 0 \\
-a^{2} 9 I & 29 D & 0 & 0 \\
0 \ldots 0 & 0 \ldots 0 & 0 \ldots 0 & 0 \ldots 0 \\
0 \ldots 0 & 0 \ldots 0 & 0 \ldots 0 & 0 \ldots 0 \\
O & O & I & 0 \\
0 \ldots 0 & 0 \ldots 0 & 0 \ldots 0 & 0 \ldots 0 \\
0 \ldots 0 & 0 \ldots 0 & 0 \ldots 0 & 0 \ldots 0 \\
O & O & O & \tilde{\varepsilon} I \\
0 \ldots 0 & 0 \ldots 0 & 0 \ldots 0 & 0 \ldots 0 \\
0 \ldots 0 & 0 \ldots 0 & 0 \ldots 0 & 0 \ldots 0
\end{array}\right) X,
\end{aligned}
$$

where $X=\left(W_{0}, \ldots, W_{N}, \Theta_{0}, \ldots, \Theta_{N}, \Phi_{0}, \ldots, \Phi_{N}\right), O$ is the zeros matrix, $I\left(n_{1}, n_{2}\right)=T_{n_{2}}\left(z_{n_{1}}\right), D\left(n_{1}, n_{2}\right)=T_{n_{2}}^{\prime}\left(z_{n_{1}}\right)$, $D^{2}\left(n_{1}, n_{2}\right)=T_{n_{2}}^{\prime \prime}\left(z_{n_{1}}\right), Y\left(n_{1}, n_{2}\right)=\left(1 / g\left(z_{n_{1}}\right)\right) I\left(n_{1}, n_{2}\right)$, $\Sigma\left(n_{1}, n_{2}\right)=f^{2}\left(z_{n_{1}}\right) I\left(n_{1}, n_{2}\right), \Omega_{0}=\chi a^{4} f^{2}\left(z_{n_{1}}\right) I\left(n_{1}, n_{2}\right)+$ $a^{2} f\left(z_{n_{1}}\right) I\left(n_{1}, n_{2}\right), \quad \Omega_{1}=\chi f^{2}\left(z_{n_{1}}\right)\left(8 D^{3}\left(n_{1}, n_{2}\right)-\right.$ $\left.4 a^{2} D\left(n_{1}, n_{2}\right)\right)-2 f\left(z_{n_{1}}\right) D\left(n_{1}, n_{2}\right)+f^{\prime}\left(z_{n_{1}}\right) I\left(n_{1}, n_{2}\right)$, $\Omega_{2}=g\left(z_{n_{1}}\right)\left(4 D^{2}\left(n_{1}, n_{2}\right)-a^{2} I\left(n_{1}, n_{2}\right)\right)+2 g^{\prime}\left(z_{n_{1}}\right) D\left(n_{1}, n_{2}\right)$, $\Omega_{3}=4 D^{2}\left(n_{1}, n_{2}\right)-a^{2} I\left(n_{1}, n_{2}\right) n_{1}=0, \ldots, N-2$ and $n_{2}=0, \ldots, N$. We computed the differentiation matrices, which are corresponded to the trail functions (5.1) analytically using Matlab routines. For more details about the Chebyshev collocation method, see [44].

We have found the solution to (5.3) is achieved very accurately by employing the $Q Z$ algorithm of Moler and Stewart [45]. This algorithm relies on the fact that there are unitary matrices $Q$ and $Z$ such that $Q A Z$ and $Q B Z$ are both upper triangular. The algorithm then yields sets of values $\alpha_{i}, \beta_{i}$ which are the diagonal elements of $Q A Z$ and $Q B Z$. The eigenvalues $\alpha_{i}$ of (5.3) are then obtained from the relation $\alpha_{i}=\alpha_{i} / \beta_{i}$, provided $\beta_{i} \neq 0$. This is very important, since the way we have constructed $B$ means it contains a singular band and we find one third of the $\beta_{i}=0$; these $\beta_{i}$ must be filtered out. Indeed, with the technique advocated here one ought always to consider the $\alpha_{i}$ and $\beta_{i}$, since as [45]. 
We have solved system (5.3) for eigenvalues $\sigma_{j}$ by using the $Q Z$ algorithm from Matlab routines. Once the eigenvalues $\sigma_{j}$ are found we use the secant method to locate where $\sigma_{j}^{R}, \sigma_{j}=\sigma_{j}^{R}+\sigma_{j}^{I}$ being the real and imaginary parts of eigenvalue $\sigma_{j}$. The value of $R$ which makes $\sigma_{1}^{R}=0, \sigma_{1}^{R}$ being the largest eigenvalue, is the critical value of $R$ for $a^{2}$ fixed. We then use golden section search to minimize over $a^{2}$ and find the critical value of $R^{2}$ for linear instability. Numerical results are reported in the next section. In our use of the Chebyshev collocation method, we used polynomial of degree between 20 and 30 . Usually 25 was found to be sufficient but convergence was checked by varying the degree by examining the convergence of the associated eigenvector (which yields the approximate associated eigenfunction).

Returning to the nonlinear eigenvalue system (4.8), the the Chebyshev collocation method yields:

$$
\begin{gathered}
\left(\begin{array}{cccc}
2 D & -I & O & O \\
B C_{1} & 0 \ldots 0 & 0 \ldots 0 & 0 \ldots 0 \\
B C_{2} & 0 \ldots 0 & 0 \ldots 0 & 0 \ldots 0 \\
\Omega_{0} & \Omega_{1} & O & R_{c} \Lambda_{22} \\
0 \ldots 0 & B C_{3} & 0 \ldots 0 & 0 \ldots 0 \\
0 \ldots 0 & B C_{4} & 0 \ldots 0 & 0 \ldots 0 \\
O & O & 2 \lambda_{1} \Omega_{2} & 0 \\
0 \ldots 0 & 0 \ldots 0 & B C_{5} & 0 \ldots 0 \\
0 \ldots 0 & 0 \ldots 0 & B C_{6} & 0 \ldots 0 \\
-R_{c} \Lambda_{21} & O & 0 & 2 \lambda_{2} L e^{-1} \Omega_{3} \\
0 \ldots 0 & 0 \ldots 0 & 0 \ldots 0 & B C_{7} \\
0 \ldots 0 & 0 \ldots 0 & 0 \ldots 0 & B C_{8}
\end{array}\right) X \\
=R_{t}\left(\begin{array}{cccc}
O & 0 & 0 & 0 \\
0 \ldots 0 & 0 \ldots 0 & 0 \ldots 0 & 0 \ldots 0 \\
0 \ldots 0 & 0 \ldots 0 & 0 \ldots 0 & 0 \ldots 0 \\
0 & 0 & \Lambda_{12} & 0 \\
0 \ldots 0 & 0 \ldots 0 & 0 \ldots 0 & 0 \ldots 0 \\
0 \ldots 0 & 0 \ldots 0 & 0 \ldots 0 & 0 \ldots 0 \\
-\Lambda_{11} & 0 & 0 & 0 \\
0 \ldots 0 & 0 \ldots 0 & 0 \ldots 0 & 0 \ldots 0 \\
0 \ldots 0 & 0 \ldots 0 & 0 \ldots 0 & 0 \ldots 0 \\
0 & 0 & 0 & 0 \\
0 \ldots 0 & 0 \ldots 0 & 0 \ldots 0 & 0 \ldots 0 \\
0 \ldots 0 & 0 \ldots 0 & 0 \ldots 0 & 0 \ldots 0
\end{array}\right) X
\end{gathered}
$$

where

$$
\begin{gathered}
\Lambda_{11}\left(n_{1}, n_{2}\right)=\left(\frac{\lambda_{1}}{g\left(z_{n_{1}}\right)}+1\right) I\left(n_{1}, n_{2}\right), \\
\Lambda_{12}\left(n_{1}, n_{2}\right)=0.5 a^{2} f^{2}\left(z_{n_{1}}\right) \Lambda_{11}\left(n_{1}, n_{2}\right), \\
\Lambda_{21}\left(n_{1}, n_{2}\right)=\left(1-\lambda_{2}\right) I\left(n_{1}, n_{2}\right), \\
\Lambda_{22}\left(n_{1}, n_{2}\right)=0.5 a^{2} f^{2}\left(z_{n_{1}}\right) \Lambda_{21}\left(n_{1}, n_{2}\right) .
\end{gathered}
$$

Then, we can determine the critical Rayleigh $R a_{E}$ for fixed $a^{2}, \lambda_{1}$ and $\lambda_{2}$. Next, we employ golden section search to minimize in $a^{2}$ and then maximize in $\lambda_{1}$ and $\lambda_{2}$ to determine $R a_{E}$ for nonlinear energy stability,

$$
R a_{E}=\max _{\lambda_{1}, \lambda_{2}} \min _{a^{2}} R^{2}\left(a^{2}, \lambda_{1}, \lambda_{2}\right),
$$

where for all $R^{2}<R a_{E}$ we have stability. The derivatives in the optimization problem are not known and so a quasiNewton (or similar) technique that does not require derivatives would have to be used. We have found the golden section search method works well on both the maximum and minimum problems. It may be a little more expensive in computer time than other techniques, but for problems like (5.5) it has been very reliable.

\section{Stability analysis results}

The numerical results are presented for $g(z)=e^{A z}, g(z)=$ $e^{B z}$, and $N_{L}=N_{U}=N_{0}$. The results in this paper are given for $N_{L}=N_{U}=N_{0}, \tilde{\varepsilon}=5$, and $\vartheta=L e=1$. This selection of parameter values was corresponds to the values in previous studies which have been deal with the problem of nonhomogeneous porosity and thermal diffusivity effects on stability and instability of double-diffusive convection $[7,8]$.

The thresholds of both the numerical linear instability and nonlinear stability results are presented in Figure 1. More comparable linear and nonlinear thresholds are apparent as the onset of convection predicted by the linear theory becomes fully stationary. However, their agreement does deteriorate as the solute Rayleigh number becomes large, indicating that the linear theory may fail to suitably emulate the physics of the onset of convection. The behaviour of the linear instability curves is in good agreement with that seen in [46]. The kink in the curves represents the point at which convection switches from steady convection $(\sigma=0)$ to oscillatory $\left(\sigma_{r}=0, \sigma_{i} \neq 0\right)$. Note that as $R_{C}$ increases the onset of convection is more likely to be via oscillatory convection as opposed to steady convection. We observe from Figure 1 that for $R_{C}=0$ there is very good agreement between the nonlinear stability and linear instability bounds. We also note that as $R_{c}$ is small the linear and nonlinear results become closer and remark that since the critical Rayleigh numbers are so close (for $R_{C}$ sufficiently small) we expect the linear analysis to have captured the essential physics of the onset of convection. However as $R_{c}$ increases the agreement between the two thresholds is not so good and we highlight this area as a region of possible subcritical instabilities.

Figure 2 explores this concept in more detail by giving a visual representation of the linear instability thresh- 


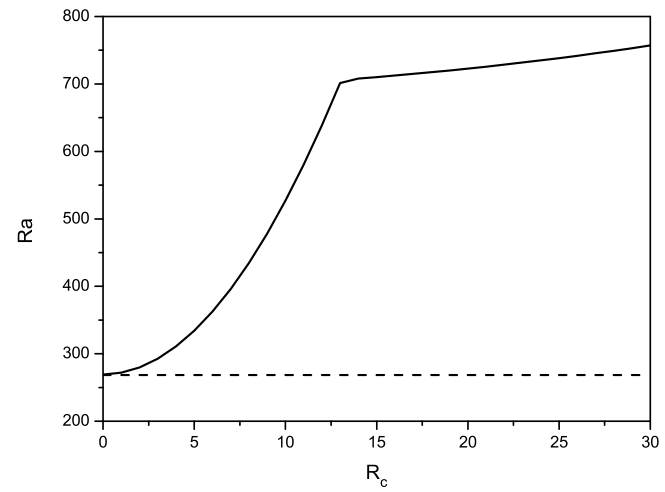

Fig. 1: Visual representation of stationary linear instability (solid line) and nonlinear stability (dashed line) thresholds, with critical Rayleigh number plotted against $R_{C}$, where $N_{0}=1, \chi=0.1$ and $A=B=1$.

olds for a variety of $\chi$ values for fixed $A, B, \varepsilon, N_{0}$ and $R_{c}$. Interesting, it is clear from figure 2 an increase in $\chi$ values causes the system to become slightly more stable. As the linear instability and nonlinear stability results clearly show slightly good agreement, we can conclude that (for the parameter ranges explored) the linear theory not accurately encapsulates the physics of the onset of convection. Thus, the novel instability results are supported by the considerable region of subcritical instabilities. However, for a fixed value of $A=B=1, N_{0}=1$ and $R_{C}=15$, we see that oscillatory convection is occurring for $\chi \leq 0.575$ and when $\chi>0.575$ we shall witness stationary convection. In Figure 3, we display the critical Rayleigh numbers

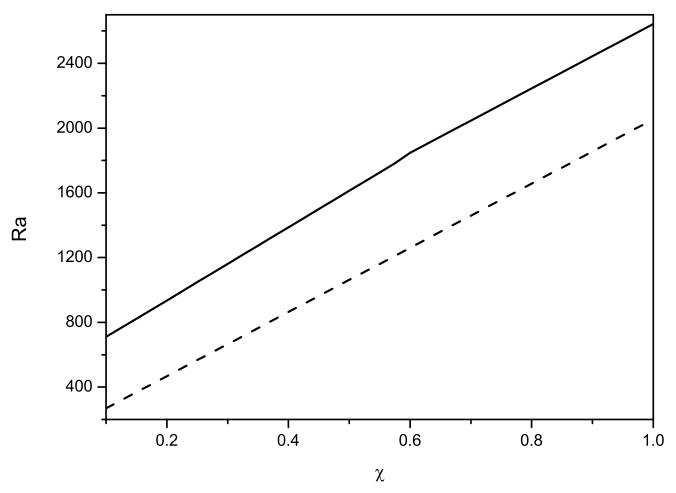

Fig. 2: Visual representation of stationary linear instability (solid line) and nonlinear stability (dashed line) thresholds, with critical Rayleigh number plotted against $\chi$, where $A=B=1, N_{0}=1$ and $R_{C}=15$. at which stability and instability begin as a function of the slip coefficient, $N_{0}$. This Figure is given for $A=B=1$, $\varepsilon=0.5, \chi=0.1$ and $R_{c}=15$. It is clear from Figure 3 that the region of potential subcritical instabilities between the linear instability and nonlinear stability thresholds is considerable. From Figure 3, we see that the oscillatory convection is always dominant in that the instability curve always lies below the stationary convection one for $N_{0} \geq 0.05$. Figure 4 shows how increasing $A$, corre-

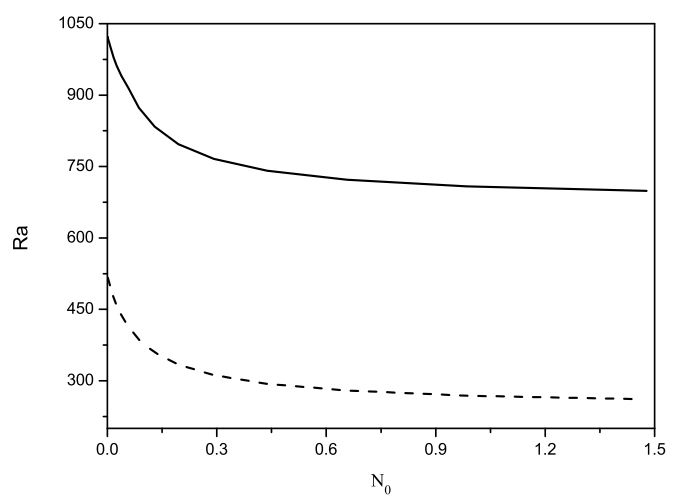

Fig. 3: Visual representation of stationary linear instability (solid line) and nonlinear stability (dashed line) thresholds, with critical Rayleigh number plotted against $N_{0}$, where $A=B=1, \chi=0.1$ and $R_{C}=15$.

sponds, to destabilization. It is clear from Figure 4 that an increase in $A$ causes the system the become more unstable, which we would physically expect. Again, it is very noticeable that the nonlinear energy stability curves are close to those of linear instability. This is reinforcing the fact that the linear curves are true representation that the physics of the onset of convection is being correctly reflected. The gap between the curves represents the small band where sub-critical bifurcation may possibly occur. For $A<-1.4$, the stationary convection become dominator in the linear instability thresholds. However, for $A \geq-1.4$, the oscillatory convection appears in the linear instability.

Figures 5 gives a visual representation of the linear instability and nonlinear stability thresholds, with critical thermal Rayleigh number $R a$ plotted against $B$. The remaining parameters are held fixed at $A=1, N_{0}=1$, $\chi=0.1$ and $R_{c}=14$. This figure shows the effect of increasing $B$ on the critical Rayleigh number. It is clear from this Figure that an increase in $B$ causes the system the become more stable, which we would physically expect. For $B<-0.1$, the stationary convection become dominator in the linear instability thresholds. When $B \geq-0.1$, 


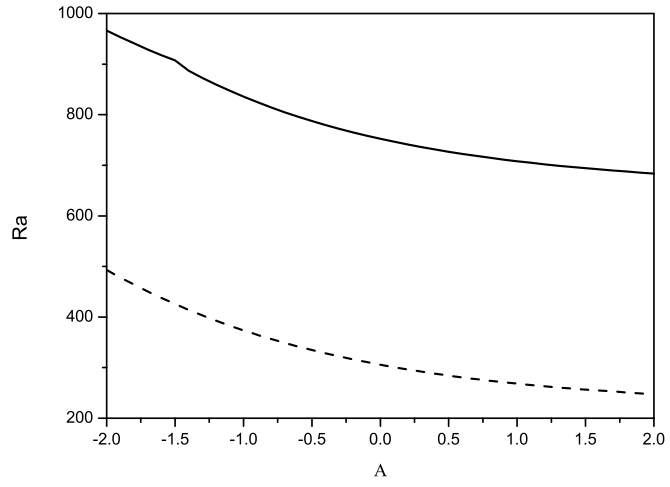

Fig. 4: Visual representation of stationary linear instability (solid line), oscillatory linear instability (dashed line) and nonlinear stability (dotted line) thresholds, with critical Rayleigh number plotted against $A$, where $B=1, \varepsilon=0.5, N_{0}=1, \chi=0.1$ and $R_{C}=14$.

the oscillatory modes become present in the linear instability thresholds. Figure 5 demonstrates that $R a$ increases with increasing $B$ which shows the stabilizing effect of $B$. It is very noteworthy that the nonlinear stability curves are close to those of linear theory. This shows that possible sub-critical instabilities may arise in a small range of Rayleigh numbers, and it also demonstrates that linear instability theory does correctly capturing the physics of the onset of convection.

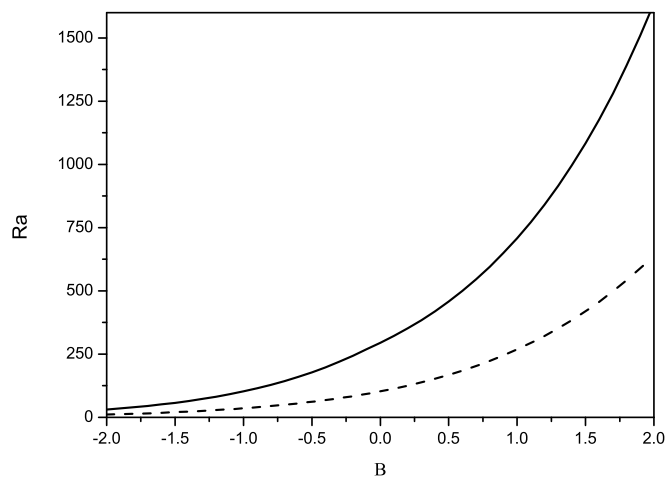

Fig. 5: Visual representation of stationary linear instability (solid line), oscillatory linear instability (dashed line) and nonlinear stability (dotted line) thresholds, with critical Rayleigh number plotted against $B$, where $A=1, N_{0}=1, \chi=0.1$ and $R_{C}=14$.

\section{Conclusions}

In this paper we have explored the effect of slip boundary conditions on the double-diffusive convection in anisotropic and inhomogeneous porous media using the Brinkman model, utilising linear stability analysis and non-linear stability by means of energy functional. A comparison between the linear stability thresholds and energy stability thresholds is made. In both cases the thermal Rayleigh number is evaluated for different combinations of the flow governing parameters. The results indicate that the increasing in the slip coefficient $N_{0}$ has a destabilizing effect in the linear and nonlinear cases. Moreover, the results show that an increase in the permeability coefficient $A$ causes a strong destabilization effect on the results. However, with increasing the thermal diffusivity coefficient $B$, the results show the stabilizing effect on the horizontal Rayleigh numbers in the linear and nonlinear cases.

\section{References}

[1] D. A. Nield, A. Bejan, Convection in Porous Media. 4th ed., Springer-Verlag, New York, (2013).

[2] B. Straughan, Stability and wave motion in porous media. Springer, Series in Applied Mathematical Sciences, vol. 165, (2008).

[3] G. Castinel, Combarnous, M.: Natural convection in an anisotropic porous layer. Int. Chem. Eng. 17, 605-614 (1977).

[4] J. F. Epherre, Criterion for the appearance of natural convection in an anisotropic porous layer. Int. Chem. Eng. 17, 615-616 (1977).

[5] O. Kvernvold, P. A. Tyvand, Nonlinear thermal convection in anisotropic porous media. J. Fluid Mech. 90, 609-624 (1979).

[6] Y. Shiina, M. Hishida, P. A. Tyvand, Critical Rayleigh number of natural convection in high porosity anisotropic horizontal porous layers. Int. J. Heat Mass Transfer 53, 1507-1513 (2010).

[7] F. Capone, M. Gentile, A.A. Hill, Convection problems in anisotropic porous media with nonhomogeneous porosity and thermal diffusivity. Acta Appl. Math. 122, 85-91 (2012)

[8] A. J. Harfash, Three-dimensional simulations for convection problem in anisotropic porous media with nonhomogeneous porosity, thermal diffusivity, and variable gravity effects. Transp. Porous Media, 102, 43-57 (2014).

[9] A. J. Harfash, Nonhomogeneous Porosity and Thermal Diffusivity Effects on a Double-Diffusive Convection in Anisotropic Porous Media International Journal of Nonlinear Sciences and Numerical Simulation 17, 205-220 (2016).

[10] C. Cercignani. The Boltzmann equation and its applications. Springer, Berlin, (1988).

[11] Z. Duan. Second-order gaseous slip flow models in long circular and noncircular microchannels and nanochannels. Microfluidics and Nanofluidics 12, 805-820 (2012). 
[12] Z. Duan and Y. S. Muzychka. Slip flow in non-circular microchannels. Microfluidics and Nanofluidics 3, 473-484 (2007).

[13] E. Lauga, M. P. Brenner, and H. A. Stone. Microfluidics: the noslip boundary condition. In C. Tropea, A. Yarin, and J. F. Foss, editors, Handbook of Experimental Fluid Dynamics, pages 1219-1240. Springer, (2007).

[14] G. L. Morini, M. Lorenzini, and M. Spiga. A criterion for experimental validation of slip - flow models for incompressible rarefied gases through microchannels. Microfluidics and Nanofluidics 1, 190-196 (2005).

[15] N. V. Priezjev. Molecular dynamics simulations of oscillatory Couette flows with slip boundary conditions. Microfluidics and Nanofluidics 14, 225-233 (2013).

[16] H. Zhang, Z. Zhang, and H. Ye. Molecular dynamics - based prediction of boundary slip of fluids in nanochannels. Microfluidics and Nanofluidics 12, 107-115 (2012).

[17] W. M. Zhang, G. Meng, and X. Wei. A review on slip models for gas microflows. Microfluidics and Nanofluidics 14, 845-882 (2012).

[18] L. P. Lefebvre, J. Banhart, and D. C. Dunand. Porous metals and metallic foams: current status and recent developments. Advanced Engineering Materials 10, 775-787 (2008).

[19] A. J. Harfash, Three dimensions simulation for the problem of a layer of non-Boussinesq fluid heated internally with prescribed heat flux on the lower boundary and constant temperature upper surface. International Journal of Engineering Science 74, 91-102 (2014).

[20] A. J. Harfash, Three dimensional simulations and stability analysis for convection induced by absorption of radiation. International Journal of Numerical Methods for Heat and Fluid Flow 25, 810-824 (2015).

[21] A. J. Harfash, Magnetic effect on convection in a porous medium with chemical reaction effect. Transp. Porous Media 106, 163-179 (2015).

[22] A. J. Harfash, A. K. Alshara, Chemical reaction effect on double diffusive convection in porous media with magnetic and variable gravity effects. The Korean Journal of Chemical Engineering 32, 1046-1059 (2015).

[23] A. J. Harfash, A. K. Alshara, Magnetic field and throughflow effects on double-diffusive convection in internally heated anisotropic porous media. The Korean Journal of Chemical Engineering 32, 1970-1985 (2015).

[24] A. J. Harfash, A. K. Alshara, A direct comparison between the negative and positive effects of throughflow on the thermal convection in an anisotropy and symmetry porous medium. Zeitschrift Fur Naturforschung A 70, 383-394 (2015).

[25] A. J. Harfash, Three dimensional simulations for convection induced by the selective absorption of radiation for the Brinkman model. Meccanica. 51, 501-515 (2016).

[26] A. J. Harfash, A. K. Alshara, On the stationary and oscillatory modes of triply resonant penetrative convection. International Journal of Numerical Methods for Heat and Fluid Flow 26, 13911415 (2016).
[27] A. J. Harfash, Resonant penetrative convection in porous media with an internal heat source/sink effect. Appl. Math. and Computation 281, 323-342 (2016).

[28] A. J. Harfash, Stability analysis for penetrative convection in a fluid layer with throughflow. International Journal of Modern Physics C 27(8), 1650101 (2016).

[29] S. Rionero, Symmetries and skew-symmetries against onset of convection in porous layers salted from above and below, International Journal of Non-Linear Mechanics 47, 61-67 (2012).

[30] S. Rionero, Absence of subcritical instabilities and global nonlinear stability for porous ternary diffusive-convective fluid mixtures. Physics of Fluids 24, 104101, (2012).

[31] S. Rionero, Triple diffusive convection in porous media. Acta Mechanica 224, 447-458 (2013).

[32] S. Rionero, Soret effects on the onset of convection in rotating porous layers via the "auxiliary system method". Ricerche di Matematica 62, 183-208 (2013).

[33] S. Rionero, Multicomponent diffusive-convective fluid motions in porous layers: Ultimately boundedness, absence of subcritical instabilities, and global nonlinear stability for any number of salts. Physics of Fluids 25, 054104, (2013).

[34] R. D. Luca, S. Rionero, Steady and oscillatory convection in rotating fluid layers heated and salted from below, International Journal of Non-Linear Mechanics, 78, 121-130 (2016).

[35] Y. Zhu and S. Granick, Limits of the hydrodynamic no-slip boundary condition, Phys. Rev. Lett. 88, 106102 (2002).

[36] J. C. Maxwell, On stresses in rarefied gases arising from inequalities of temperature, Phil. Trans. Roy. Soc. Lond. 170, 231-256 (1879).

[37] E. P. Muntz, Rarefied-gas dynamics, Ann. Rev. Fluid. Mech. 21, 387-417 (1989).

[38] V. S. J. Craig, C. Neto and D. R. M.Williams, Shear-dependent boundary slip in an aqueous Newtonian liquid, Phys. Rev. Lett. 87, 054504 (2001).

[39] C. Neto, V. S. J. Craig and D. R. M. Williams, Evidence of sheardependent boundary slip in Newtonian liquids, Eur. Phys. J. E 12, S71-S74 (2003).

[40] Y. Zhu and S. Granick, Rate-dependent slip of Newtonian liquid at smooth surfaces, Phys. Rev. Lett. 87, 096105 (2001).

[41] C.L.M.H. Navier, Mémoire sur les lois du mouvement des fluides. Mémoires de l'Acad'emie Royale des Sciences d l'Institut de France 6, 389-440 (1823).

[42] B. Straughan, The energy method, stability, and nonlinear convection, Applied Mathematical Sciences, second ed., vol. 91, Springer, 2004.

[43] S. Chandrasekhar, Hydrodynamic and hydromagnetic stability. Dover, New York (1981).

[44] A. J. Harfash, Numerical methods for solving some hydrodynamic stability problems. International Journal of Applied and Computational Mathematics 1, 293-326 (2015).

[45] C. Moler and G. Stewart, An algorithm for generalized matrix eigenproblems. SIAM. J. Numerical Anal. 10, 241-256 (1973).

[46] D. D. Joseph, Uniqueness Criteria for the Conduction-Diffusion Solution of the Boussinesq Equations. Arch. Rational Mech. Anal. 35, 169-177 (1969). 